\title{
Simulated Worlds: Relaying HPC knowledge at secondary schools
}

\section{Dr. Rüdiger Berlich ${ }^{1}$}

Gemfony scientific UG (haftungsbeschränkt);

Formerly Steinbuch Centre for Computing (SCC) of Karlsruhe Institute of Technology (KIT)

Karlsruhe, Germany

E-mail: r.berlichegemfony.eu

\section{Thomas Gärtner}

Steinbuch Centre for Computing (SCC) of Karlsruhe Institute of Technology (KIT)

Karlsruhe, Germany

E-mail: gaertnerekit.edu

\section{Jörg Hilpert}

Department of Technology and Environmental Sociology (SOWI V), University of Stuttgart Stuttgart, Germany

E-mail: joerg.hilpertasowi.uni-stuttgart.de

\section{Peter Lürßen}

Bismarck Gymnasium ("secondary school”),

Karlsruhe, Germany

E-mail: Luerssen@bismarck-gymnasium.de

\section{Leticia Wilke}

Institute for High Performance Computing (IHR), University of Stuttgart

Stuttgart, Germany

E-mail: wilkedihr.uni-stuttgart.de

Simulierte Welten (engl. "Simulated Worlds") relays HPC knowledge at secondary schools in the German state of Baden Wuerttemberg, through the collection, design and provision of related material, the documentation of successful teaching concepts, as well as direct interaction with teachers and scholars. The project was initiated by High Performance Computing Centre Stuttgart (HLRS) and Steinbuch Centre for Computing (SCC) in Karlsruhe, and enjoys support from the Department of Technology and Environmental Sociology (SOWI V) of University of Stuttgart. The team, consisting of both scientists and teachers, particularly focuses on computational simulations. This area can also be used as a vehicle to relay the strong (but frequently invisible) impact that HPC applications have on society today. This paper focuses on the practical aspects of teaching the complexities of computational simulations at schools, analyzing the boundary conditions and presenting the chosen strategy.

ISGC 2013; March 17-22, 2013, Academia Sinica, Taipei, Taiwan

$1 \quad$ Speaker 


\section{Introduction}

We live in a two-tiered world. One side is moved by simulations ranging from testing the best automobile design, to developing and testing the highly advanced medication and surgical tools, all calculated over many hours on incredibly fast and expensive supercomputers. On the other side is the general public, including young students going through their high-school years, wondering if the mathematics and physics they have learned will ever be used for anything they can think of as useful.

These two worlds exist in parallel to each other: the scientists working in the high performance computing centers around the world probably do not think too often about their high school days; and rarely will teenagers sitting in a classroom even notice all the simulations already embedded into their lives every day. The same applies to their parents and indeed most people in our society.

At first glance, these two groups do not seem to be relevant to each other, since students will go on through school, being prepared for life. And those scientists will go on through their professional lives, developing their theories and bringing in further advance in technology. However, these two worlds could only profit from a stronger interaction with one another.

This paper describes how 3 major science and education institutions in the German state of Baden Württemberg joined efforts, and started a science outreach project that would work as a bridge to connect those two worlds, and create a steady and clear stream of communication between them. The aim of the project "Simulated Worlds" was to reach out to students and get them actively involved in the workings of high performance computing through simulations, raising awareness to the importance of that kind of technology to modern society.

The project was born out of the necessity to make High Performance Computing a science more known to the public in general. Simulations, in turn, grow in importance every day and we rely on them more as we need to have so many things tested in a safer and less expensive way and develop them more efficiently. The cost of such efficiency is high and grows accordingly to the developments made in that sector. Due to the variety of possible topics and the direct impact on society, simulations initially appeared as a useful vehicle to relay the importance of HPC. However, over the course of the project, the topic of "computational simulations" gained a life on its own, putting it at the focus of the project and indeed giving it its name.

Most citizens have no idea of the amount of public money invested into Simulations and HPC and should be made aware of the need of such investments. The next challenge was to find a way to execute the plan. What would be the best route?

\section{Why high school?}

Many of the science outreach programs in Germany are oriented towards particularly gifted students, beyond the High School level. Finding partners for those projects in science and industry is relatively easy, since many groups are interested in disseminating their projects and finding high potentials. 
However, the abundance of such projects casts doubts as to the usefulness of the approach. While the identification of particularly skilled individuals certainly needs to be part of the agenda (compare section VIII), a limitation to this group also limits the scope of the project, which indeed wants to reach as far an audience as is possible, considering all constraints.

The team thus decided to work with a group of regular schools, initially aiming for the average high school student, starting around the age of 14 . This decision supports the idea of mingling natural sciences with the average population; after all, the majority is average, and it is the majority we would like to reach. Also, while gender matters naturally need to be taken into account, the project cannot solve all underlying problems. In fact, the project would like to encourage groups, which are as mixed as possible, mirroring the way society itself works. The material developed in the project, however, has to be application-centric and is thus inherently agnostic to gender issues.

\section{Why HPC and simulations?}

It is indeed easy to find good reasons why the topics of HPC and simulations should be taught at schools. To name but a few:

- Many far-reaching decisions in science, technics and economy are taken on the basis of HPC and computational simulations. Yet there is a striking gap between visibility of these technologies and their impact on society. And while their advantages can be easily described (just think of the life-saving aspects of many simulations), their inherent dangers and risks are far more complex and subtle. Yet, it is the youngest in our society that will experience the largest impact of computational simulations. Gaining an understanding of both the pros and cons might thus even become a pre-requisite to a self-determined life one day.

- Even when discounting any direct impact on scholars, the funds going into the design and operation of HPC installations as well as their applications warrant public interest in the topic.

- And finally, while our society increasingly depends on HPC and simulations to answer the question "what, if?" the percentage of scholars from the "generation iPad" actually being exposed to HPC technology will gradually decrease. An outreach program designed to increase interest in the topic can thus contribute to the long-term survival of this discipline.

So the idea is not only to make high performance computing visible to students and awaken their curiosity and maybe even turn them into future professionals in this field, but to get them engaged in the discussions, involving them as citizens in the social side of the matter:

Can simulations be used for everything? How far should we rely on them? Are they worth the costs they generate? What are the opportunities and risks arising from this technology in my own life? And should I care?

\section{Key messages}

The answer to the last question, as perceived by the project team, is of course clear. And so the first key message is: "Yes, if we want to actively shape our future, we undoubtedly need 
computational simulations and HPC as a means of performing this work; and the need for these techniques will only increase over time". An understanding of the importance of the topic can be given simply by providing examples for the broad range of applications to scholars. This can be done on all levels of prior knowledge and technical interest.

It is even easier to summarize the most important risk of computational simulations. Indeed this can be done in two words: "wrong conclusions". The reasons for these, however, are varied and warrant a more in-depth analysis.

- Simulations represent a complex model of reality, and their development will be expensive. Some areas will be modeled overly perfect, discounting second- and third-level influences on purpose. For other areas, these influences might not even be known. And while a given simulation will likely give good results for the original intended use, it can be assumed, that they might also be used to answer questions from border cases, not at the core of the original design. This will happen particularly often, if giving a proper answer would require an expensive redesign or re-calibration of the code, or if time is scarce and answers are needed quickly (“do we need to evacuate?"). In the absence of any other information, such possibly flawed answers will then be taken as the basis of decisions.

- It is up to the author of a simulation to decide, which parts of reality are modeled, and which are not. The decision will be driven by his or her personal level of knowledge, and by project constraints. Decisions will thus be subjective and necessarily error-prone.

- Complex programs have bugs. Bug hunting can be done on different effort levels, but will ultimately be limited by project resources. The point in time when a program is declared to be production code will ultimately be determined by the question, whether it gives the expected results. However, there is a very high level of subjectivity in what an expected result actually is. What if reality, as is so often the case, shows unexpected behavior? Then the simulation will give bad answers, even if it seemingly gives the correct answer.

- The results of some simulations cannot be checked easily, be it because doing so is too expensive, or because they make predictions reaching far into the future. We do need such answers, however we should be very aware that these answers could be flawed.

- Only an expert will understand most (but never all) aspects of a given model of reality. However, particularly for larger simulations, there will be a fluctuation of personnel. If a program is used for a number of years, it might well be different people drawing conclusions from a simulation than those who have created the initial model.

- Reality will be described by observations, and measurements, as well as rules derived from this data. These measurements and observations (and thus the derived rules) can be wrong, rendering the entire simulation useless. Sometimes there is no (economical) way to discover this situation.

None of the topics on this list is easy to teach, particularly if one has to rely on the mere presentation of examples. It will thus usually be necessary for students to practically explore the difficulties by creating their own (simple) simulations. A comparison with real measurements will then help to discover the deficiencies of a given model of reality. Targeting this at high school students has several additional advantages: 
- At that age the pupils are easier to engage in projects in which they can work on their own, without permanent teacher control.

- Parents are still involved in discussions and projects, thus making them part of it. High school students can be better prepared for a dialogue with scientists.

- Pupils get an early glimpse into the professional life of scientists, opening a door for them to explore a possible career path for themselves.

\section{Participants}

Another precondition to the success of such teaching efforts is access to dedicated technical knowledge, as well as direct insights in the teaching practices at schools. The project is one of many funded by the Ministry of Science, Research and the Arts Baden-Württemberg (MWK), involving three other German institutions:

- HLRS High Performance Computing Centre Stuttgart: bearing with "Hermit" one of the fastest civil super-computers in Europe, devoted to scientific projects and industrial development. At the time of writing it was in the Top-30 of the Top 500 list.

- Steinbuch Centre for Computing (SCC) at Karlsruhe Institute of Technology (KIT) hosts the German Tier-1 center in the worldwide EGI grid and is also the location of another (at the time of writing) system in the Top 500 list.

- Institute of Social Sciences of Stuttgart University: Department of Environmental and Technological Sociology ("SOWI V"). It is part of a project responsible for raising awareness in the community, of the importance of their opinion and involvement in the doings of natural sciences.

Jointly, HLRS and SCC have a solid base in technology research and comprise a large user base active in the field of computational simulations. Researchers with dedicated technical knowledge are thus comparatively easily accessible. Where a particular field of knowledge is not covered, it is expected that contacts can be established to one of the projects funded by MWK or other German funding agencies. SOWI-V contributes the procedural knowledge and sociological insights into the German educational system.

\section{The HPC ambassador}

During the first attempts to relay HPC knowledge at schools, it became quickly clear that the project would profit immensely from the direct participation of dedicated teachers as part of the project team. The project team has thus created the position of "HPC Ambassador" in cooperation with MWK. At the time of writing, a single teacher fills this role, with 1-2 other expected to join the team in the near future. The project funds their activities so that their work becomes part of their daily duties at their home schools. The HPC ambassador is the first point of contact for new teachers interested in the material and methods developed by Simulated Worlds. He also participates in all strategic decisions and the development of new content, making sure that it fits the needs of schools. 


\section{Simulations and curricula}

The topic of computational simulations is indeed not entirely alien to curricula in German schools; however, it rarely features prominently. Thus, when designing a new program destined to indeed relay dedicated technical and sociological information to scholars, one needs to ask why this hasn't happened so far to a much larger extent.

There is a number of possible explanations:

- A dominant reason of course lies in the complexity of the topic. The foundations of HPC alone as an "enabling technology" are challenging. Simulations however, are even more complex, given the huge variety of possible applications, each of which has its own depths. This is even truer as few scholars will have in-depth experience in programming. And even the authors of a particular simulation themselves will rarely be able to understand all possible aspects of their programs. So how can this be expected from students (and teachers) alien to the subject?

- Questions answered by simulations - at least beyond the ubiquitous climate prediction - can be very special. Except for some lighthouse projects it will thus be hard to convince the audience that a particular example will have a direct impact on their lives.

- HPC and computational simulations have gained their overarching importance only over the past few years, hand in hand with the increase of our computing capabilities. Thus teachers being capable of relaying this knowledge will either need to regularly attend trainings or will otherwise tend to be young. So only a portion of all teachers in the field will be capable of teaching the topic.

The impact of a perceived lack of teachers with a computer sciences background at schools in Germany - the role is frequently filled by teachers with a natural sciences background - is in contrast considered to be small by the project team. After all, it is not the goal of the project to teach scholars detailed techniques for the creation of production-level simulations, but rather to teach them the complexities of the overall field. For this, it seems far more important for teachers to understand particular fields of applications. Biology or physics teachers, who also teach computer sciences, are well suited for this role.

Still, the project needs to make the training of teachers one of its prime objectives.

\section{The knowledge pyramid}

Based on the analysis and means presented in the previous sections, the project team has designed a strategy, shown figuratively in figure 1.

The project continuously gathers information about particular application scenarios. These are categorized and made available for passive consumption (i.e. consumption without the necessity for the interaction with the project team) through the project web page and an E-Learning system (ILIAS). Such information might comprise examples for concrete simulations, presentations, opinions provided by experts or simple sample simulations.

The usage of an E-Learning system makes it possible to allow interested teachers and even students to contribute, correct and expand the available material. The offer is not only targeted at students, but at the general public. 


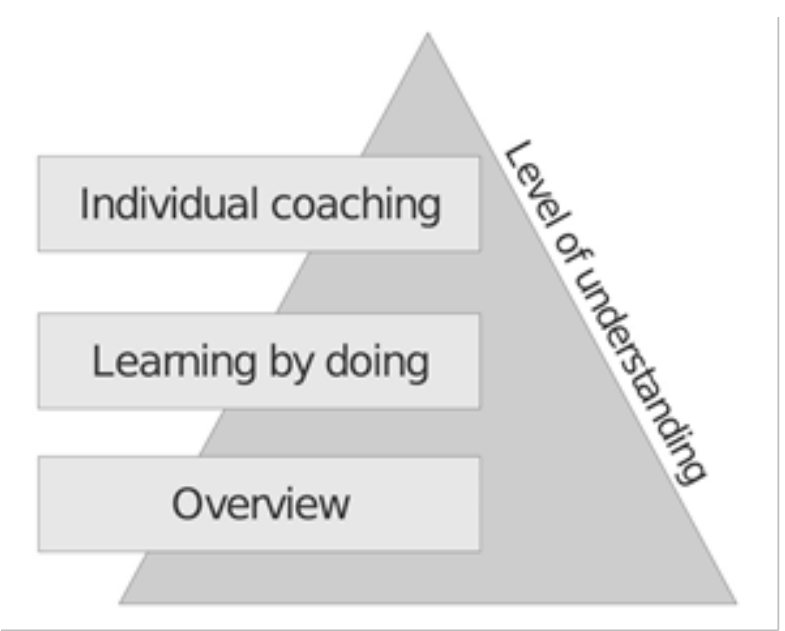

Figure 1: The project tries to relay knowledge on three levels, by passive provision of data, through courses and by personal coaching of particularly gifted scholars.

It should be noted, that the project started with the development of concrete courses, on the second level of the pyramid. Hence, at the time of writing, the collection of material is still far from satisfactory. Nevertheless it is expected, that new course material can be extracted from the collection, once it is more complete.

Evening lectures at interested schools throughout the state of Baden Württemberg give interested teachers, parents and students a first opportunity to directly interact with project and possibly initiate a later cooperation. These lectures were already held successfully in a number of cases, and individual reactions of people attending the events indicated that the chosen topic ("Simulation and Reality"), centered on opportunities and risks of computer-based simulations, was met with interest by the audience.

The second level of the knowledge pyramid shown in figure 1 - "Learning by doing comprises the direct interaction with groups of students. These are tasked with the creation of small, self-contained simulations, giving them an opportunity to develop own ideas in a controlled environment.

The initial assumption "teachable" content would have to be largely of a non-technical nature proved incorrect in first courses held by the project in close cooperation with a local school Bismarck Gymnasium in Karlsruhe.

In the context of a joint participation in the "city of young scientists" event in Karlsruhe, altogether 16 students from 7th to 10th grade developed simple simulations using the programming language "Scratch".

Scratch comprises many aspects of a real-world programming language (if-statements, loops and even simple multi-threading), but allows assembling programs from simple "building blocks" with mouse. This avoids any syntax error and thus limits mistakes to logical errors. Programs can be executed immediately, and may even include simple sound and graphics.

Students thus had the "opportunity" to make the same logical mistakes observed in large-scale simulations (compare section IV), but were relieved from many of the more arcane burdens of programming. 
The students had to develop a simple billiard games, comprising 6 holes and two balls. The resulting simulation thus had to take into account the momentum transfer between two balls, as well as the interaction with the banks of the table. No trigonometric functions (which were still unknown to some students) had to be known. However, the energy loss of the balls while traversing the table had to be taken into account.

No interaction between more than two balls was required, but was indeed implemented by a team of older students. All work had to be done at home, with preparation and discussion happening in three double-hours. Finally, the results of the simulation had to be compared with a real billiard game.

\section{Making mistakes}

It was indeed one of the goals of the procedure that students made mistakes. Despite the relative simplicity of the topic, programs quickly grew large and exhibited all aspects discussed above in section IV. For example, the students programs did not comprise a possible spin applied to the balls in reality, neither could they take into account imperfections of the real-world billiard game. And of course the functions used for the energy loss were far from perfect. It was the stated goal of this part to let students learn by example, which succeeded well. Simulation and reality of the billiard game did differ, and sometimes did so to a large extent.

From this perspective, it was then easy for students to understand that very similar issues arise with the most complex of simulations, which was the core theme of the session.

Further examples were provided, such as an egg rolling down an incline. The comparison between simulation and reality proved interesting in this case.

The work was finally presented in the summer of 2012 during the "festival of young scientists" in Karlsruhe, Germany.

\section{Energy efficiency}

Simulated Worlds cooperates with a project that has developed a game called "ENERGETIKA". With it, students may design the energy mix of a medium-sized country (with Germany being the obvious prototype), without bringing about upheaval or environmental catastrophes. The game itself can be understood as a simulation, albeit with many interactive aspects. It allows relaying an understanding of the processes implemented in larger, real-world simulations, particularly those designed to make predictions about the results of specific scenarios in societies. Students successfully identified some of the challenges involved by playing this game and analyzing the results of specific scenarios (e.g. very conservative or very "green" choices).

\section{Model schools}

Both for the accompanying teacher - now the first HPC ambassador of the project - and the project team itself, the work with the students was very time-consuming. For a small project such as "Simulated Worlds", it is not possible to do the same for all interested schools throughout Baden-Württemberg. 
Hence the idea is to develop such material in close cooperation with a small number of 3-4 "model schools" and to make the results available through the E-Learning system.

A possible second project phase after the formal end of the funding period in the second quarter of 2014 would then have to concentrate on "business development" and an evolutionary improvement of the material being developed. The focus of the current project phase, however, lies on the creation of a tried and tested framework, so that interested teachers will not have to start from scratch (no pun intended).

The results of this strategy discussion were also presented to students and teachers alike in the context of focal groups.

\section{Direct coaching}

Coaching of high-potentials forms the final level of the "Knowledge Pyramid" (compare figure 1). The overall idea of the project is to allow the general public, including interested students and teachers, to inform themselves about the topic of computational simulations. Then more indepth knowledge can be taught to students at selected schools. Finally, the most interested individuals (i.e. the "high-potentials") are invited to participate in real-world HPC projects during their final year at school.

This procedure was put into practice in the spring and summer of 2012, with 6 selected students during their final year at school. They worked on concrete projects in HPC, ranging from Big Data to a portal for the creation of X509 certificates. The focus went beyond the topic of simulations and was targeted at work usually found at HPC centers. Experts of the corresponding field coached the students.

\section{Science Café}

Other activities comprise a "Science Cafe", being held in the spring of 2013 for the first time. Here, groups of students prepare questions around a particular topic related to HPC and simulations. Then an expert from the field is invited for a prolonged Q\&A session.

\section{Finding partners}

Finding schools to work with was a harder task to complete than forming a concept. We approached the schools explaining the Simulated Worlds, exposing the concept and offering a role in the project. The results were very disappointing: most schools did not reply to our call, very few teachers were present in workshop we offered and even fewer accepted to work with us. Of the hundred schools approached, initially only two enlisted to work with us as model schools in our.

The appointment of the HPC ambassador and the exploitation of many personal contacts changed this for the better. The project now believes to have a sufficient number of model schools for the successful completion of the current project phase.

In the end, success in this matter boiled down to being able to identify the right contacts, as is the case practically everywhere in real life. 


\section{Conclusion}

The topic of "computational simulations" has so many aspects that its relevance to society, including its opportunities and risks, cannot be fully explored without direct interaction with the topic.

Relaying this knowledge at schools was initially assumed to be limited to showing examples for simulations, as it was assumed that scholars would not have sufficient interest and capability to develop custom simulations.

With the help of the programming language Scratch and in close cooperation with a model school, however, a complete teaching unit could be developed.

The three-tiered design of the project (compare figure 1) proved to be very useful. The approach can be likened to a filter, where general knowledge is relayed in the first tier (quite possibly also to the general public, so it fits in nicely with general dissemination and outreach activities), interested groups of scholars are taught specifics in the second tier (and teachers are coached to relay existing material or further develop own ideas), and the most talented individuals may receive direct coaching in the third tier.

A project such as "Simulierte Welten" would not be possible without direct interaction of the project team with teachers, scholars and even parents. Calibration of the initial plans through direct feedback of the "targets" of ones efforts is an important precondition for success.

The appointment of "HPC ambassadors" (i.e. dedicated teachers knowledgeable both in the intricacies of relaying complicated technical facts to the youngest of our society, as well as, through their education, with an advanced understanding of the topic at hand) proved to be the most important decision taken by the project so far. HPC ambassadors serve as a kind of bridge between the specialized knowledge of scientists and practitioners on the one hand, and scholars on the other.

With many surrounding offers, such as a science cafe, evening lectures and further seminars, and based on the experiences made so far, the project team believes to be capable of fulfilling its task: to design a program that allows to relay the topics of HPC and computational simulations at schools in the German state of Baden Württemberg.

\section{Acknowledgements}

The authors would like to thank the Ministry of Science, Research and Art of the German state of Baden-Württemberg for their kind support of the project, as well as all teachers and scholars that have been directly or indirectly involved in the project up to date. We would also like to thank the scientists and practitioners of SCC, HLRS and SOWI V for their dedicated and generous support of this project. 